\title{
Dissipation in ultra-thin current-carrying superconducting bridges; evidence for quantum tunneling of Pearl vortices
}

\author{
F. Tafuri ${ }^{1}$, J.R. Kirtley ${ }^{2}$, D. Born ${ }^{3}$, D. Stornaiuolo ${ }^{3}$, P.G. Medaglia ${ }^{4}$, P. Orgiani ${ }^{4}$, G. Balestrino ${ }^{4}$, V.G. Kogan ${ }^{5}$ \\ ${ }^{1}$ INFM-Coherentia Dip. Ingegneria dell'Informazione Seconda Universitá di Napoli, Aversa (CE), Italy \\ ${ }^{2}$ IBM Watson research center, Route 134 Yorktown Heights, NY 10598 \\ 3 INFM-Coherentia, Dipartimento di Scienze Fisiche, Universitá di Napoli "Federico II" Napoli, Italy \\ 4 INFM-Coherentia, Dipartimento di Ingegnaria Meccanica, Universitá di Roma Tor Vergata, Roma, Italy \\ ${ }^{5}$ Ames Laboratory - DOE and Department of Physics and Astronomy, Iowa State University, Ames IA 50011-3160
}

(Dated: August 15, 2018)

\begin{abstract}
We have made current-voltage (IV) measurements of artificially layered high- $T_{c}$ thin-film bridges. Scanning SQUID microscopy of these films provides values for the Pearl lengths $\Lambda$ that exceed the bridge width, and shows that the current distributions are uniform across the bridges. At high temperatures and high currents the voltages follow the power law $V \propto I^{n}$, with $n=\Phi_{0}^{2} / 8 \pi^{2} \Lambda k_{B} T+1$, and at high temperatures and low-currents the resistance is exponential in temperature, in good agreement with the predictions for thermally activated vortex motion. At low temperatures, the IV's are better fit by $\ln V$ linear in $I^{-2}$. This is expected if the low temperature dissipation is dominated by quantum tunneling of Pearl vortices.

PACS numbers: $74.50 .+\mathrm{r}, 74.78 . F k, 74.78 .-\mathrm{w}$
\end{abstract}

Soon after the seminal work of Caldeira and Leggett 1], the possibility of quantum tunneling of vortices in thin films was considered by Glazman and Fogel 2]. This idea was studied extensively in theory and experiment in relation to finite flux-creep rates in hard type-II superconductors for $T \rightarrow 0$. For thermally activated creep, the rates should go to zero in this limit, see review 3 and references therein. Within creep models, the barriers through which the vortices tunnel are due to material disorder and are treated statistically.

Narrow current carrying thin-film bridges offer a unique opportunity to study both thermal activation and quantum tunneling through the well defined potential barriers that are tunable by current and temperature. Within the London approach, the barrier shape is well known for bridges narrow relative to the Pearl length $\Lambda=2 \lambda^{2} / d$, where $\lambda$ is the penetration depth and $d$ is the film thickness.

To realize these conditions we use ultra-thin high- $T_{c}$ films with extremely large Pearl lengths $\Lambda \sim 100 \mu \mathrm{m}$. The critical temperature of these films $T_{c} \sim 40 \mathrm{~K}$ provides a relatively easy $T$-domain to work in, and the data reported are quite robust.

When current $I$ flows through the bridge at high temperatures, vortices are thermally activated at the strip edges and pushed in, causing dissipation and a non-zero voltage $V$. We show that these processes should lead to a power-law dependence $V \propto I^{n}$ with the exponent $n$ determined by the film parameter $\Lambda$ and temperature:

$$
n=\frac{\phi_{0}^{2}}{8 \pi^{2} \Lambda(T) k T}+1
$$

( $\phi_{0}$ is the flux quantum and $k$ is the Boltzmann constant). This is confirmed by the high-temperature IV's and by the independently measured Pearl length.
However for low T's, the IV's show a slower current dependence than required by thermal activation. Instead, a quantum tunneling model utilizing the ideas of Refs. 1, 2, along with our knowledge of the barrier shape, provide a good representation of the data.

In the present work, ultrathin sandwiches of $\left[\mathrm{Ba}_{0.9} \mathrm{Nd}_{0.1} \mathrm{CuO}_{2+x}\right]_{5} /\left[\mathrm{CaCuO}_{2}\right]_{2} /\left[\mathrm{Ba}_{0.9} \mathrm{Nd}_{0.1} \mathrm{CuO}_{2+x}\right]_{5}$ (CBCO 5/2/5) were used. They consist of one superconducting infinite layer block (two $\mathrm{CaCuO}_{2}$ unit cells), sandwiched between two charge reservoirs (5 Ba-based unit cells). The structure was grown on (001) $\mathrm{SrTiO}_{3}$ substrates, with nominally zero miscut angle, by using a focussed $\mathrm{KrF}$ excimer pulsed laser source [4, 5]. The films were photolithographically patterned into bridges. In this paper we present measurements from a "wide" bridge approximately $85 \mu \mathrm{m}$ across. After measurement, this bridge was narrowed by a factor of 2 and then remeasured. The patterning of such a thin layer $(5 \mathrm{~nm}$ with a superconducting cell only $1 \mathrm{~nm}$ thick) protected by a $100 \mathrm{~nm}$ amorphous cap layer required specially careful photolithography and milling. The use of a cap layer deposited in situ required pre-deposited $\mathrm{Au}$ contacts (details will be given elsewhere). Four terminal measurements of the current-voltage characteristics were realized down to $300 \mathrm{mK}$ in an Oxford Heliox probe suitably modified by superconducting wiring to minimize heating at elevated currents. The bridges were imaged with a scanning SQUID microscope (SSM) [6, 7] to determine current uniformity and the film Pearl length.

Figure 17 shows an SSM image of the wide bridge after cooling in a $22 \mathrm{mG}$ field. The bridge outline is visible along with trapped Pearl vortices [8, [9]. Fig. 10 shows a cross-section through one Pearl vortex, after recooling in a smaller field to reduce the density of vortices. Fitting such cross-sections results in low temperature Pearl 
(a)

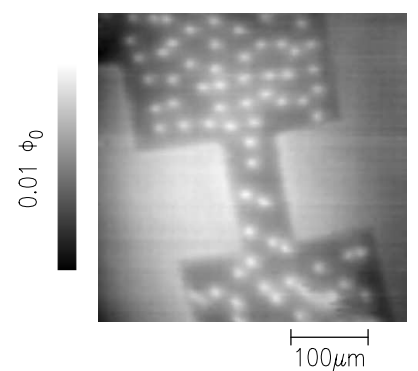

(c)

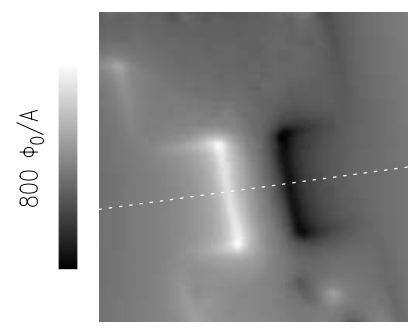

(b)

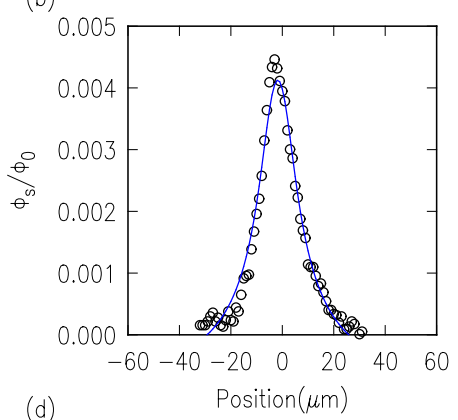

(d)

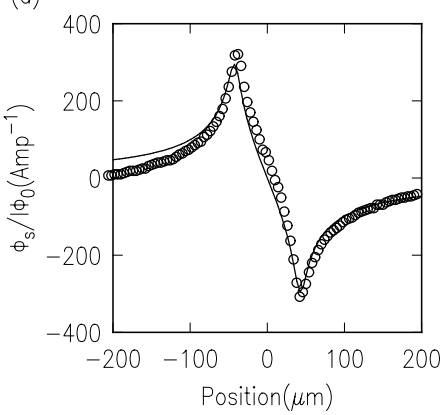

FIG. 1: (Color online) (a) SSM image of the wide $5 / 2 / 5$ CBCO bridge after cooling in a $22 \mathrm{mG}$ field at $5 \mathrm{~K}$ with an $8 \mu \mathrm{m}$ square pickup loop. (b) Cross-section through an isolated Pearl vortex after cooling in a $1 \mathrm{mG}$ field. The line fit is obtained using the field distribution $h_{z}(x, z)$ given in Ref. 9, with an effective pickup loop height $z=5 \mu \mathrm{m}$ and $\Lambda=400 \mu \mathrm{m}$. (c) SSM image with a $104 \mathrm{~Hz}, 100 \mu \mathrm{A}$ r.m.s. a.c. current passing through the bridge at $T=20 \mathrm{~K}$. (d) Crosssection through the data along the dashed line in (c); the solid line is calculated for uniform current through the bridge, with $z=5 \mu \mathrm{m}$.

lengths of $400 \pm 50 \mu \mathrm{m}$ for this bridge. Fits to scanning susceptibility measurements of the same bridge gave $\Lambda=$ $200 \pm 20 \mu \mathrm{m}$ (see Ref.9 for details of these procedures). This difference may be due to the finite lateral dimensions of the film, which are not accounted for in the modelling. The length $\Lambda$ is therefore longer than the bridge width, and we expect the current through the bridge to be uniform, as confirmed by the SSM image and fit of Fig.11,d. The field $h_{z}$ (normal to the film) is maximum near the edges $x=0, W$ where vortices or antivortices nucleate, penetrate the strip by being pushed in by the Lorentz force $\phi_{0} I / c W$, and annihilate near the strip middle. We focus on the barrier for vortices; the one for antivortices is obtained by replacing $x$ with $W-x$.

For narrow strips of width $W \ll \Lambda$ with no current, the energy of a vortex at a position $0<x<W$ is [10]:

$$
\epsilon(x)=\frac{\phi_{0}^{2}}{8 \pi^{2} \Lambda} \ln \left(\frac{2 W}{\pi \xi} \sin \frac{\pi x}{W}\right),
$$

$\xi$ is the coherence length. The vortex energy in the presence of a uniform current is

$$
U(x)=\epsilon(x)-\frac{\phi_{0} I}{c W} x .
$$

The current causes suppression of the barrier maximum $U_{m}$ and pushes the maximum position $x_{m}$ from the middle (at $I=0$ ) toward the edge $x=0$. We obtain after simple algebra: $x_{m}=(W / \pi) \tan ^{-1}\left(I_{0} / I\right)$ and

$$
\begin{array}{r}
U_{m}=\epsilon_{0}\left(\ln \frac{2 W}{\pi \xi \sqrt{1+I^{2} / I_{0}^{2}}}-\frac{I}{I_{0}} \tan ^{-1} \frac{I_{0}}{I}\right), \\
I_{0}=\frac{c \phi_{0}}{8 \pi \Lambda}, \quad \epsilon_{0}=\frac{\phi_{0}^{2}}{8 \pi^{2} \Lambda} .
\end{array}
$$

For $\Lambda \approx 400 \mu \mathrm{m}, I_{0} \approx 2 \times 10^{-3} \mathrm{~mA}$ at low temperatures and decreases on warming. For the majority of our data $I / I_{0} \gg 1$ and Eq. (4) simplifies to:

$$
U_{m}=\epsilon_{0} \ln \frac{2 W I_{0}}{e \pi \xi I},
$$

where $e=2.718$. The logarithmic dependence of the barrier height on the current results in power-law IV's, the dependence encountered in models of vortices moving through random potentials in disordered materials [3]. In our case, it is caused by a well-defined barrier.

If vortices cross the strip via a thermally activated process, a voltage $V \propto \exp \left(-U_{m} / k T\right)$ is generated, i.e.,

$$
\begin{aligned}
& V \propto \exp \left(-\frac{\epsilon_{0}}{k T} \ln \frac{2 W I_{0}}{e \pi \xi I}\right)=\left(\frac{I}{I_{d}}\right)^{m}, \\
& m=\frac{\epsilon_{0}(T)}{k T}=\frac{\phi_{0}^{2}}{8 \pi^{2} \Lambda(T) k T}, \\
& I_{d}=\frac{c \phi_{0}}{8 e \pi^{2} \lambda^{2} \xi} W d .
\end{aligned}
$$

where $I_{d}$ is of the order of the bridge depairing current. Thus, the model provides not only the power-law per se, but gives the exponent in terms of the film parameters. We note also that our model differs from that used in Ref. 11 to interpret similar data.

As is usually the case with thermal activation, it is difficult to fix the "attempt frequency" in the probability $\exp \left(-U_{m} / k T\right)$. Still, the number of vortices penetrating the strip must be proportional to the field value at the edges, i.e., to the current $I$. Adding a prefactor const. $I$ in Eq. (7), we obtain the exponent $n=m+1$ of Eq. (1) which gives the correct limit for $T \rightarrow T_{c}$.

The upper panel of Fig.2 shows IV measurements for the wide bridge on a log-log scale. All curves are nearly straight (except noisy low-voltage parts), i.e., $V \propto I^{n}$ at high currents. The exponent $n$ extracted from powerlaw fits to the data is shown in the lower panel, for both the wide and narrow bridges. The solid line is $n(T)$ of Eq. (1), in which the two-fluid $\Lambda=2 \lambda_{0}^{2} / d\left(1-t^{4}\right)$ has been used for simplicity; $t=T / T_{c}$. Fitting the high- $T$ part of the data for the wide bridge we obtain $T_{c} \approx 38 \mathrm{~K}$ and $\Lambda(0) \approx 320 \mu \mathrm{m}$ in agreement with $\Lambda(0) \approx 200-400 \mu \mathrm{m}$ independently measured by SSM. Thus, the thermal activation model works well above $\approx 15 \mathrm{~K}$, where it gives 

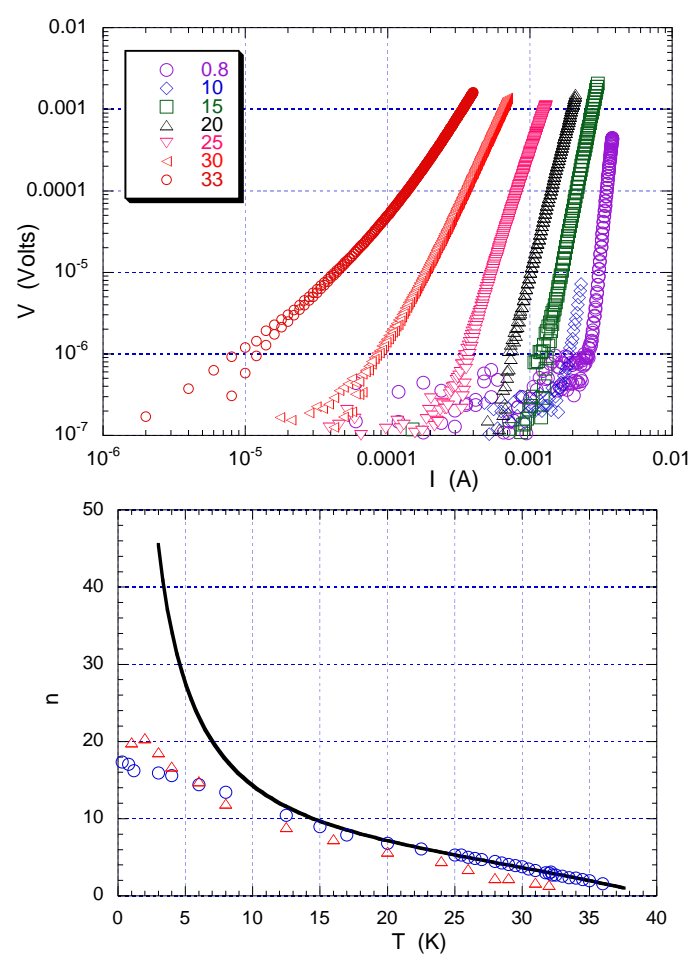

FIG. 2: (Color online) The upper panel: $V(I)$ on a log-log scale at temperatures indicated in the legend. Lower panel: The exponent $n$ extracted from $V \propto I^{n}$ for the wide bridge (circles) and the narrow bridge (triangles). The solid line is $n(T)$ of Eq. (1) with with the best-fit parameters $T_{c}=37.6 \mathrm{~K}$ and $\Lambda(0)=317 \mu \mathrm{m}$.

correct values of the exponent $n(T)$. Although $T_{c}$ of the narrow bridge was slightly lower after repatterning, the high temperature exponents were quite close, which means that the Pearl length was nearly the same in both bridges.

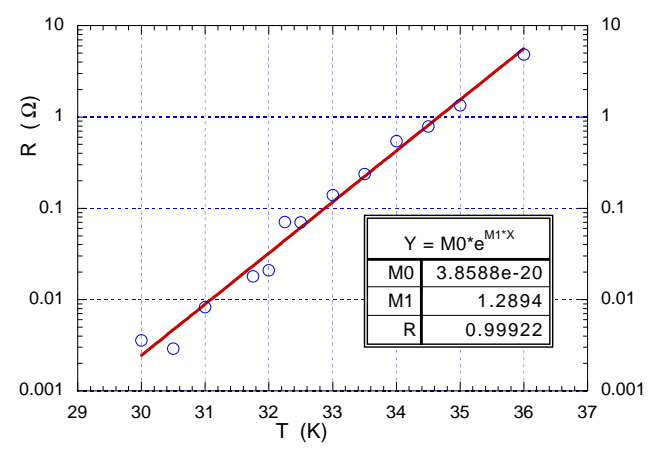

FIG. 3: (Color online) $R(T)$ for $30<T<36 \mathrm{~K}$ and fit to an exponential dependence.

Another quantity which can be extracted from the IV's is the low-voltage differential resistance, defined as $R=$ $(d V / d I)_{I \rightarrow 0}$. At low temperatures, the voltages at low currents are exceedingly small and cannot be extracted from the noise. However, reliable determination of $R$ is possible above roughly $30 \mathrm{~K}$. Fig. 3 shows that in this domain $R \propto \exp \left(T / T_{0}\right)$.

To understand this result, we turn again to the voltage expression (7) for thermally activated vortex motion. As mentioned above, the prefactor in this expression is proportional to the current because the number of vortices attempting to overcome the barrier is proportional to the edge fields, i.e., to $I$. Therefore, calculating $R=(d V / d I)_{I \rightarrow 0}$, we can leave the current dependence only in the prefactor and set $I=0$ in $\exp \left[-U_{m}(I) / k T\right]$. We then have:

$$
U_{m} \approx \epsilon_{0} \ln \frac{2 W}{\pi \xi}=\frac{4 \phi_{0}^{2}(1-t)}{8 \pi^{2} \Lambda(0)} \ln \frac{2 W}{\pi \xi},
$$

where $\Lambda(0)$ is the low temperature Pearl length, and the two-fluid approximation $\Lambda=\Lambda(0) /\left(1-t^{4}\right) \approx \Lambda(0) / 4(1-$ $t)$ is again used. Then, the voltage and the resistance near $T_{c}$ should be proportional to

$$
\exp \left(+\frac{\phi_{0}^{2} T}{2 \pi^{2} \Lambda(0) k T_{c}^{2}} \ln \frac{2 W}{\pi \xi}\right) .
$$

Thus, we expect the behavior $R \propto \exp \left(T / T_{0}\right)$ with

$$
T_{0}=\frac{2 \pi^{2} \Lambda(0) k T_{c}^{2}}{\phi_{0}^{2} \ln (2 W / \pi \xi)}
$$

where $T_{0}$ depends weakly on $T$ via $\ln \xi$. Note that our model does not allow us to literally take the limit $T \rightarrow T_{c}$ because the energy expression (2) is valid only for $W \gg \xi$. The data of Fig. [3] correspond to $T_{0} \approx 0.78 \mathrm{~K}$. Expression (12) yields a smaller value $T_{0} \approx 0.3 \mathrm{~K}$.

We stress that $R \propto \exp \left(T / T_{0}\right)$ is obtained here for the thermally activated process, so that this dependence per $s e$ is not necessarily a manifestation of quantum tunneling (compare with 12]).

At low temperatures, the thermal activation model fails. The fit values for $n(T)$ in Fig. 2] do not diverge as thermal activation requires [13]. Hence, we turn to the possibility of quantum tunneling. According to Ref. 2, the tunneling probability for overdamped processes is proportional to $\exp \left(-\gamma \eta x_{b}^{2} / \hbar\right)$ where $\gamma$ is a geometric factor related to the barrier shape, $\eta$ is the drag coefficient, and $x_{b}$ is the barrier width. Unlike thermal activation, for which only the barrier height is relevant, for the tunneling the barrier width dominates the probability.

Since the potential $U=0$ [14] at the position of vortex entry, the width $x_{b}$ is a root of $U(x)=0$, or of

$$
\ln \left(\frac{2 W}{\pi \xi} \sin \frac{\pi x}{W}\right)=\frac{I}{I_{0}} \frac{\pi x}{W} .
$$

As argued above, $I / I_{0} \gg 1$; therefore $\pi x / W$ must be small. Since the $\log$ is a slow function, we have

$$
x_{b} \approx C_{0} \frac{W I_{0}}{\pi I}
$$


where $C_{0}$ is a constant of the order one. Hence, the barrier width shrinks with increasing current. One can show by solving numerically Eq. (13) that for $I / I_{0} \sim 10^{3}$ (the range of our data) $C_{0} \approx 5$.

We then expect the voltage for high currents to behave according to

$$
\ln V=C_{1}-C_{2} \frac{\eta W^{2}}{\hbar} \frac{I_{0}^{2}}{I^{2}}
$$

Here, $C_{1}$ is a constant related to the attempt frequency, whereas $C_{2}=C_{0}^{2} \gamma / \pi^{2} \sim 2.5 \gamma$.

In Fig. 4 we plot $\log V$ for a few $T$ 's versus $I^{-2}$ for both wide and narrow bridges. Clearly, the straight parts of the IV's for low temperatures and high currents are in agreement with Eq. (15). With increasing T, the IV's curve away from a straight line much faster, signalling deviations from predominantly quantum tunneling. We fit the data for $0.8 \mathrm{~K}$ of the wide bridge and for $1 \mathrm{~K}$ of the narrow one to $V=V_{0} \exp \left(-\alpha / I^{2}\right)$ to obtain $V_{0}=0.43 \mathrm{~V}$, $\alpha=1.0 \times 10^{-4} \mathrm{~A}^{2}$ for the first and $V_{0}=0.97 \mathrm{~V}, \alpha=0.14 \times$ $10^{-4} \mathrm{~A}^{2}$ for the second.

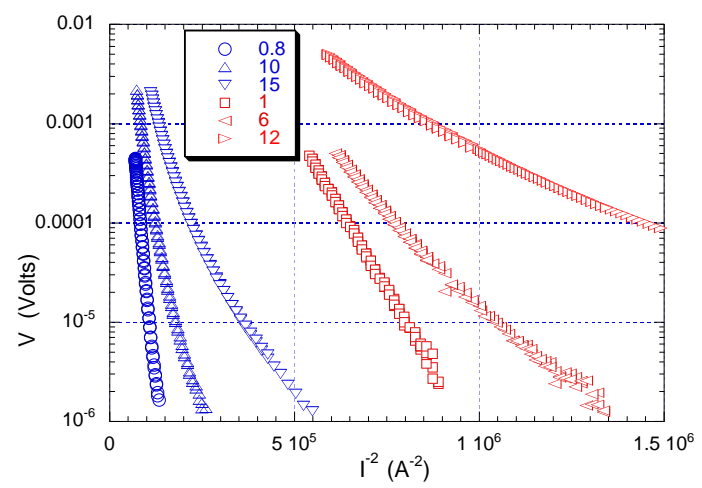

FIG. 4: (Color online) The voltage $V$ versus $1 / I^{2}$ for temperatures indicated in the legend. The left group of IV's is for the wide bridge with $W=85 \mu \mathrm{m}$; the right group is for $W=42.5 \mu \mathrm{m}$.

Going back to Eq. (15) for the quantum tunneling, we note that the coefficient of $I^{-2}$ is proportional to $W^{2}$. Therefore, as an extra check of applicability of this equation, one can compare $\alpha$ 's of the two bridges. Given the approximate character of our model, the reduction of $\alpha$ by a factor of 7 in the bridge $W / 2$ as compared with the expected reduction by a factor of 4 , shows that quantum tunneling is consistent with our data.

Moreover, the pre-exponential factor $V_{0}$ proportional to the attempt frequency should go as $1 / W$ because as magnetostatics shows - the edge field is $\propto 1 / W$. The ratio of $V_{0}$ 's for the two bridges is $0.97 / 0.43=2.3$ instead of the expected factor of 2 . This is yet another indication that the low- $T$ data are consistent with the tunneling model.

Further quantitative tests of the quantum tunneling model would be possible if the drag coefficient $\eta$ and the barrier shape factor $\gamma$ were well known. Unfortunately, this is not the case. The factor $\gamma=\pi / 2$ was found for a barrier $U(x)$ as a cubic parabola [15], quite different from our case. For $I_{0}=2.5 \mu \mathrm{A}$ and $\mathrm{W}=85 \mu \mathrm{m}$, we estimate $\eta \gamma \sim 10^{-16}$ CGS. If one takes $\gamma \sim 1$, this gives the drag coefficient about two orders of magnitude smaller than the Bardeen-Stephen value $\eta=\phi_{0}^{2} d / 2 \pi \xi^{2} c^{2} \rho_{n}$. KramerPesh contraction [16] of the vortex core at low $T$ 's might be a cause of the $\eta$ suppression.

During this work, we have also searched for a Berezunskii-Kosterlitz-Thouless transition in the domain of the power-law IV's. We, however, did not detect any discontinuities in the $T$ dependence of $n$. We believe this is because in bridges with $W<\Lambda$, interaction of vortices is short-range with a cutoff at distances $W$.

To summarize, at high temperatures we observe the power-law IV's well described by thermally activated motion of vortices through the thin-film strips narrow on the scale of Pearl $\Lambda$ for which the shape of the potential barrier is well established. The predicted dependence of the exponent $n$ on $\Lambda(T)$ is in good agreement with the data. The low temperature data are consistent with the overdamped quantum tunneling of Pearl vortices, the size of which in our films is indeed macroscopic since $\Lambda \sim 0.1 \mathrm{~mm}$.

The authors thank L. Bulaevskii for thoughtful remarks. This work has been supported by ESF projects "Pi-Shift" and "QUACS", and by MIUR program "Quantum effects in Nano-structures and Superconducting devices".

[1] A.G. Caldeira and TA.J. Leggett, Phys. Rev. Lett. 46, 211 (1981).

[2] L.I. Glazman and N.Ya. Fogel, Sov. J. Low temp. Phys. 10, 51 (1984); Fiz. Nizk. Temp. 10, 94 (1984).

[3] G. Blatter et al., Rev. Mod. Phys. 66, 1125 (1994).

[4] G. Balestrino et al., Phys. Rev. B 62, 9835 (2000).

[5] G. Balestrino et al., Appl. Phys. Lett. 79, 99 (2001).

[6] J.R. Kirtley et al., Appl. Phys. Lett. 66, 1138 (1995).

[7] J.R. Kirtley et al., Appl. Phys. Lett. 74, 4011(1999).

[8] J. Pearl, Appl. Phys. Lett. 5, 65 (1964).

[9] F. Tafuri et al., Phys. Rev. Lett. 92, 157006 (2004).

[10] V.G. Kogan, Phys. Rev. B 49, 15874 (1994). The part of this work dealing with vortex in a half-plane film is erroneous. The energy arguments and the treatment of vortices in samples small on the scale $\Lambda$ are correct. The energy expression (2) has been confirmed by J.R. Clem, unpublished 1996, and by G.M. Maximova, Sov. Phys. Solid State, 40, 1607 (1998).

[11] J.M. Repaci et al., Phys. Rev. B 54, 9674 (1996).

[12] Y. Liu et al., Phys. Rev. Lett. 68, 2224 (1992).

[13] Taking a faster than the two-fluid low- $T$ behavior of $\Lambda$ (for materials with gap nodes) would have made the increase of $n(T)$ at low $T$ 's even stronger. The levelling of $n(T \rightarrow 0)$ is not pronounced in other reports, see e.g. Ref.11. 
[14] One can take the vortex energy at $x \sim \xi$ near the edge, $\epsilon(\xi)=\epsilon_{0} \ln 2$, as the starting energy. This results only in a renormalization of the large constant $2 W / \pi \xi \rightarrow W / \pi \xi$, but leaves the structure of Eq. (13) unchanged.

[15] A.I. Larkin, Yu.N. Ovchinnikov, JETP Lett. 37, 3821
(1983).

[16] W. Pesh and L. Kramer, J. Low Temp. Phys. 15, 367 (1974). 


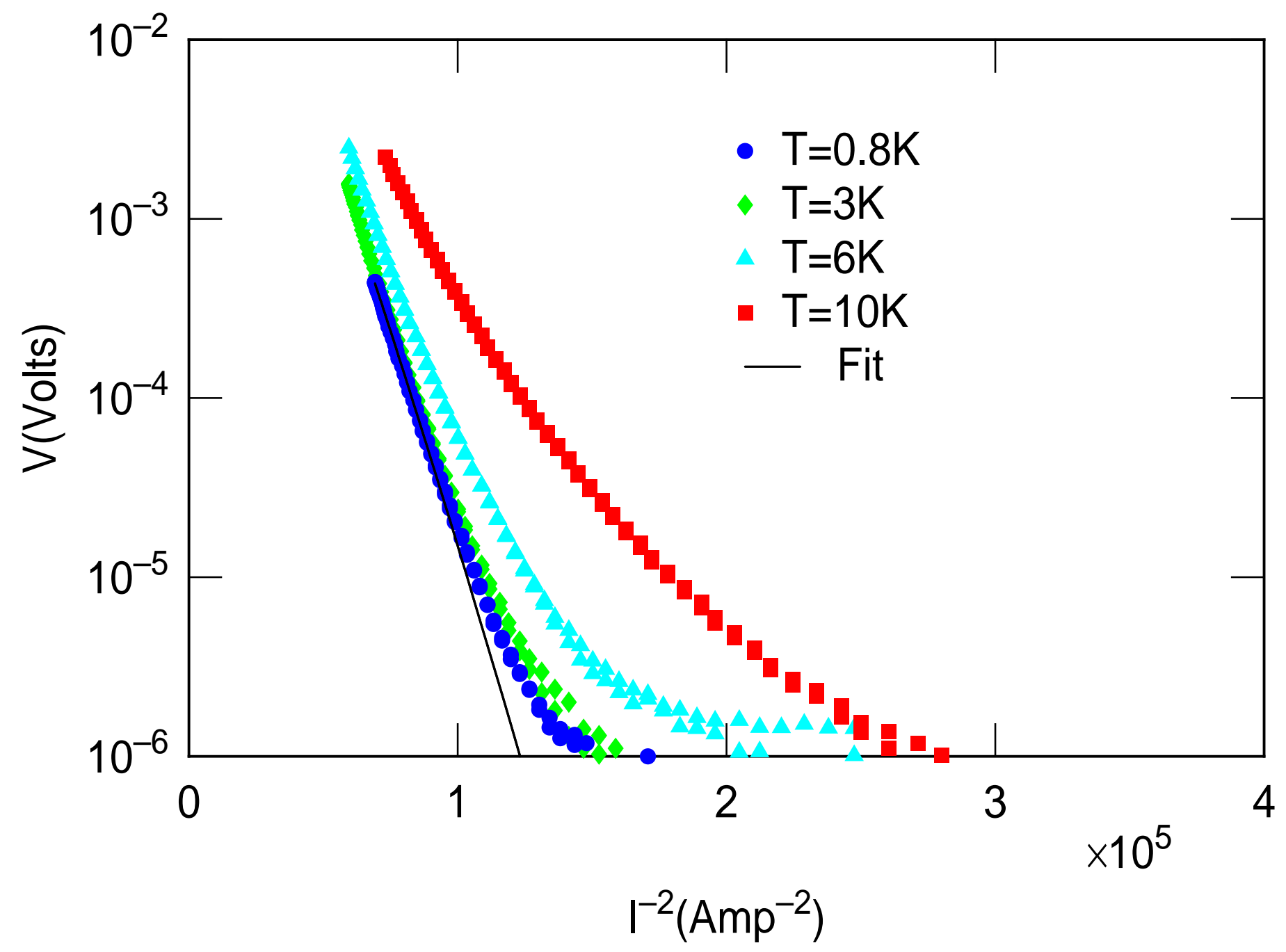




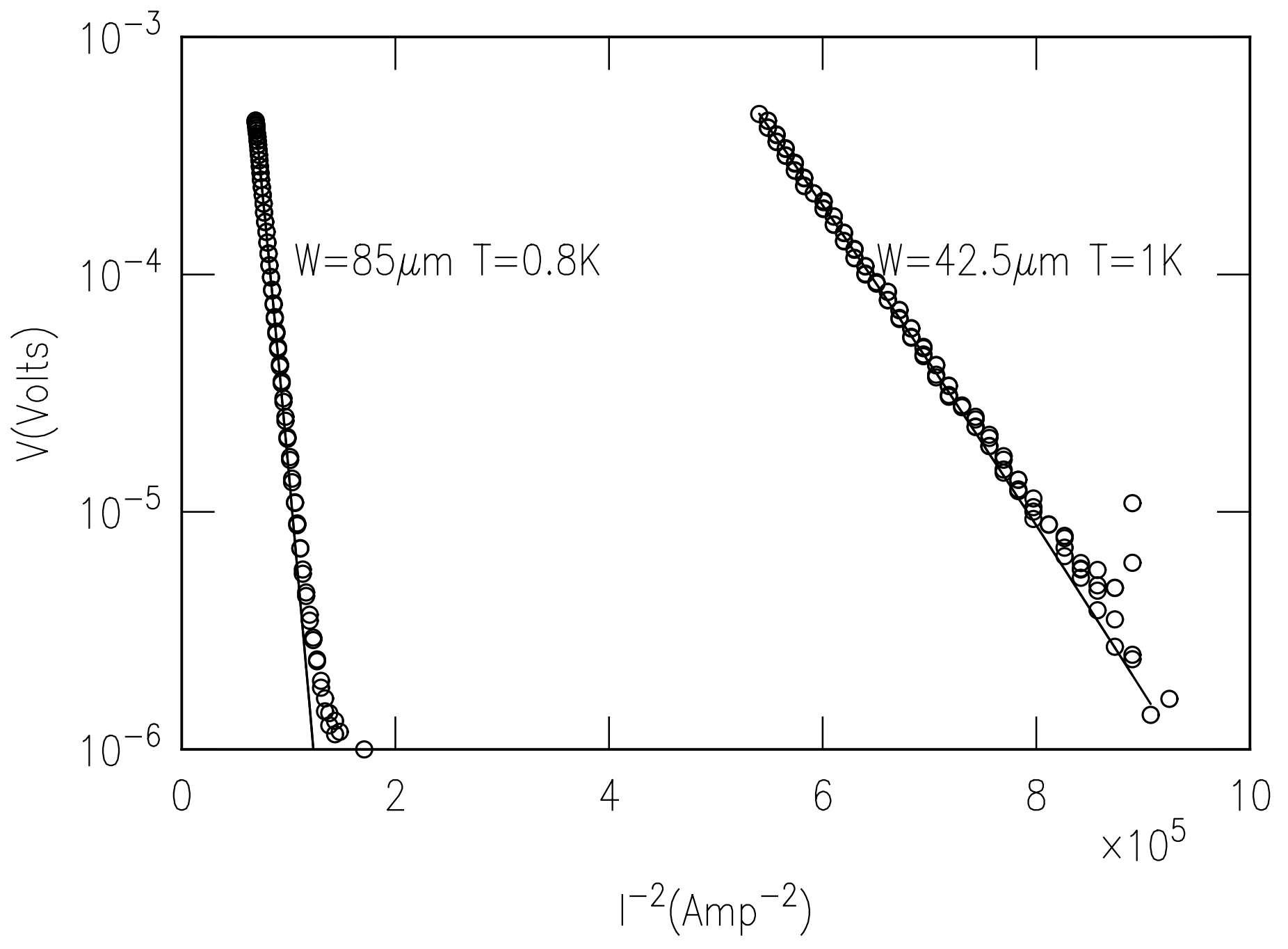

